\title{
ASOCIACIONES DE BIVALVOS DEL TRIÁSICO EN ESPAÑA
}

\author{
Ana MÁRQUEZ-ALIAGA ${ }^{1}$ y Vicente MARTÍNEZ ${ }^{1}$ \\ ${ }^{1}$ Dpto. de Geología, Universitat de València. C/Dr. Moliner, 50. 46100 Burjassot (Valencia).
}

\begin{abstract}
Márquez-Aliaga, A. y Martínez, V. 1996. Asociaciones de bivalvos del Triásico en España. [Bivalve assemblages of Triasic System in Spain]. Revista Española de Paleontología, N Extraordinario, 103-113. ISSN 0213-6937.

\section{ABSTRACT}

During the Triassic the Iberian Peninsula was placed at the western part of the Tethys Sea. The Middle-Late Triassic (AnisianNorian) rocks of the Iberian, Catalonian and External Betic (Prebetic and Subbetic) Ranges, contain three main intervals interpreted as prograding carbonate ramps: lower Muschelkalk (Anisian), upper Muschelkalk (Ladinian) and Imon Dolomites Formation (Norian); some carbonatic levels appear in the Subbetic (Carnian). The fossil record is frequently scarce and bad preserved. During one century (1853-1954), spanish paleontological works on the Muschelkalk dealt with several punctual aspects and showed taxonomical confusion. In this paper the results of the last ten years of paleontological research on triassic bivalves from Spain have been updated. At the moment, one Anisian, three Ladinian, two Ladinian-Carnian, one Carnian and one Norian bivalve assemblages have been identified. The record appears in sediments from protected areas of shallow carbonatic ramps. The bivalve species have not biostratigraphical meaning but its assemblages characterize each carbonatic intercalation. The most of the taxa are common in the Tethys realm.
\end{abstract}

Keywords: Bivalves, Triassic, Muschelkalk, Iberian Peninsula, Paleontological synthesis.

\section{RESUMEN}

Durante el Triásico la Peninsula Ibérica formaba parte del extremo más occidental del Tethys. En los sedimentos del Triásico Medio-Superior (Anisiense-Noriense) de las Cordilleras Ibérica, Catalana y Béticas externas (Prebético y Subbético) se pueden diferenciar, en ocasiones, hasta tres niveles carbonatados: Muschelkalk inferior (Anisiense), Muschelkalk superior (Ladiniense) y las Dolomías de la Formación Imón (Noriense), y algunas intercalaciones carbonáticas en el Subbético (Carniense). El registro fósil es escaso y está mal conservado. Durante un siglo (1853-1954), los trabajos paleontológicos sobre el Muschelkalk español se referían a aspectos puntuales y muestran una gran confusión en la sistemática. En este trabajo se sintetizan los resultados obtenidos en la investigación paleontológica de los bivalvos triásicos de España durante los últimos diez años. Por el momento, se han podido diferenciar varias asociaciones características: una en el Anisiense, tres en el Ladiniense, dos en el Ladiniense-Carniense, una en el Carniense y una en el Noriense. El registro de bivalvos está relacionado con ambientes protegidos de rampas carbonatadas someras. Las especies de bivalvos no tienen significado bioestratigráfico de pequeño rango pero sus asociaciones permiten caracterizar las diferentes intercalaciones carbonatadas. La mayoría de los taxa son comunes en la cuenca del Tethys.

Palabras clave: Bivalvos, Triásico, Muschelkalk, Península Ibérica, Síntesis paleontológica.

\section{INTRODUCCIÓN}

Los estudios de Paleontología en nuestro país se vienen desarrollando durante los últimos años con gran intensidad. Sin embargo, llama la atención la baja proporción en España de paleontólogos del Triásico. La razón de este abandono puede ser considerada desde varias vertientes, pero existió un "a priori" que queda perfectamente enmarcado en las palabras del gran paleontólogo español Mallada (1880), cuando en su Sinopsis sobre especies fósiles de España, Sistema Triásico, dice: "los fósiles escasean extraordinariamente por todas partes y no suelen ser de fácil determinación los moldes que los representan". Se trata de un momento delicado para la historia de la vida. Tras la gran extinción del final del Pérmico la diversidad biótica sufrió una enorme merma. El bajo registro fósil mundial del Triásico marino es más marcado si se compara con el de otras épocas. Este es el caso del registro fósil en los extensos afloramientos del Triásico de la Península Ibérica, los cuales, paleogeográficamente, formaban parte del extremo más occidental del Tethys, correspondiendose sus unidades litoestratigráficas con ambientes marinos restringidos. Entre otras, estas consideraciones explicarían la baja diversidad específica antes aludida. Por otro lado, existen diversos aspectos tafonómicos para explicar la mala preservación de los registros fósiles del Triásico marino español.

Entre los macrofósiles, son los moluscos los mejor representados, especialmente los bivalvos. Ello se pone de manifiesto en el trabajo regional sobre el Triásico de los Catalánides realizado por Virgili (1958). En él, por primera vez, se engloba una síntesis paleontológica en su contexto estratigráfico y se tienen en cuenta todos los estudios puntuales que, sobre varios grupos, habían realizado distintos autores. Sin embargo, tanto en la Cordillera Ibérica, como en las Béticas externas (Prebético y Subbético) o las islas Baleares, las diversas atribuciones específicas, del contenido paleontológico de distintos afloramientos del Muschelkalk, sufren un contínuo cambio aleatorio. Entre otros autores, cabe citar a Verneuil y Collomb (1853); Cortázar(1875, 1885); Dereims (1898); Würm (1911, 1913); Darder (1914); Vilaseca (1920); Sos (1933); Schmidt (1935) y Bataller (1954), quienes durante un siglo (1853-1954) apor- 
taron conocimiento al Triásico español. La característica general de estos trabajos es la naturaleza puntual de las distintas observaciones. Además, la ausencia de estudios regionales no permite integrar los conocimientos paleontológicos en su contexto estratigráfico. Sin embargo, la diversidad obtenida para los bivalvos triásicos, basada en sus publicaciones, era relativamente alta. Se diferenciaban 140 taxones distintos, y este aspecto no se hallaba en concordancia con el planteamiento expresado por Mallada (1880) al que ya hemos aludido.

A mediados de los años setenta se retoma el estudio sistemático de los bivalvos triásicos de España (Márquez-Aliaga, 1976), pero existía ya una concepción de la paleontología que suponía un cambio de mentalidad científica, a la hora de abordar los múltiples problemas que conlleva la interpretación de los registros fósiles. La sistemática se vería afectada en la misma línea. Es por ello que, metodológicamente, nos impusimos para el citado estudio varios presupuestos, algunos de los cuales fueron discutidos por Márquez-Aliaga y De Renzi (1984) y que sintetizamos como: 1.- Una revisión bibliográfica exhaustiva de los antecedentes, analizando la justificación taxonómica de cada especie. 2.- La búsqueda de nuevos materiales, con el levantamiento previo de numerosas columnas estratigráficas. 3.- La revisión de las distintas colecciones de fósiles españoles, depositadas tanto en museos nacionales como extranjeros. 4.- El estudio comparativo de nuestras faunas con las de otras cuencas geográficas próximas. 5.- El estudio tafonómico de los registros fósiles estudiados, en su doble vertiente bioestratigráfica/fosildiagenética. 6.- La integración de los fósiles en su contexto paleobiológico.

Los dos últimos aspectos metodológicos son los que consideramos más importantes. En primer lugar, porque suponen un cambio de mentalidad en la interpretación de los fósiles, la cual estaba basada en una concepción de taxonomía tipológica. En segundo lugar, porque son fundamentales para llegar a conclusiones de índole paleoecológica y bioestratigráfica.

Basándonos en estas consideraciones, se comenzó el estudio sistemático detallado de los bivalvos del Triásico Medio del Sector Meridional de la Cordillera Ibérica y de las Cordillera Costero Catalanas; en este caso se revisó la colección de fósiles (clasificados por el Dr. Villalta) utilizada por Virgili (1958) en el apartado paleontológico del Triásico de los Catalánides. El material de la Cordillera Ibérica, procede, en su gran mayoría, de nuevos hallazgos (colecciones A. Márquez y A. Goy) y su estudio sistemático constituyó el elemento central de la tesis doctoral de Márquez-Aliaga (1976, 1983, 1985). Durante la década posterior se ha seguido avanzando en el conocimiento de los bivalvos del Triásico español con el hallazgo de numerosos especímenes. Por otra parte, el estudio que se viene realizando en grupos de fósiles triásicos con mayor valor bioestratigráfico -como ammonites (Goy, 1986, 1995), foraminíferos (Márquez, 1994) y conodontos (March, 1991) - y el avance de los conocimientos estratigráficos en distintos dominios de la Peninsula Ibérica y Baleares -en la Cordillera Ibérica (López-Gómez y Arche, 1994; García-Gil, 1994; Pérez-Arlucea y Rey, 1994), en las Cordilleras Costero Catalanas (Calvet y Marzo, 1994), en Menorca (Llompart et al, 1987), en las Béticas (Martín-Algarra, 1987; Fernández et al, 1994) y en los Pirineos (Calvet et al, 1994a)-, nos está permitiendo la interpretación de los bivalvos, dentro de su peculiaridad como "fósiles de facies", como verdaderos indicadores paleoambientales. Actualmente, el conocimiento integrado del registro fósil marino español nos permite avanzar en interpretaciones paleogeográficas más concretas (Hirsch y Márquez-Aliaga,1988). Así, con respecto a los sedimentos, el Triásico de la Peninsula Ibérica es de "Facies Germánica" pero con respecto a su componente faunístico se halla integrado en la Cuenca del Tethys (Budurov et al, 1993).

\section{CORDILLERA IBÉRICA}

En la Cordillera Ibérica (Fig. 1) se han podido identificar bivalvos en tres tramos carbonatados. Los primeros se corresponden con los dos niveles marinos del Muschelkalk, equivalentes al M-1 y M-3 de Virgili (1958) en las Cordilleras Costero Catalanas. En el sector meridional, por la presencia de dos transgresiones marinas en el Triásico Medio, ha sido denominado "Trías de Tipo Mediterráneo". En el sector nor-occidental, por la presencia única de la última transgresión marina, ha sido denominado "Trias de Tipo Ibérico" (Virgili et al, 1977; Sopeña et al, 1983). El tercer tramo se corresponde con la última transgresión marina del final del Triásico Superior (Formación Imón) (Goy et al, 1976).

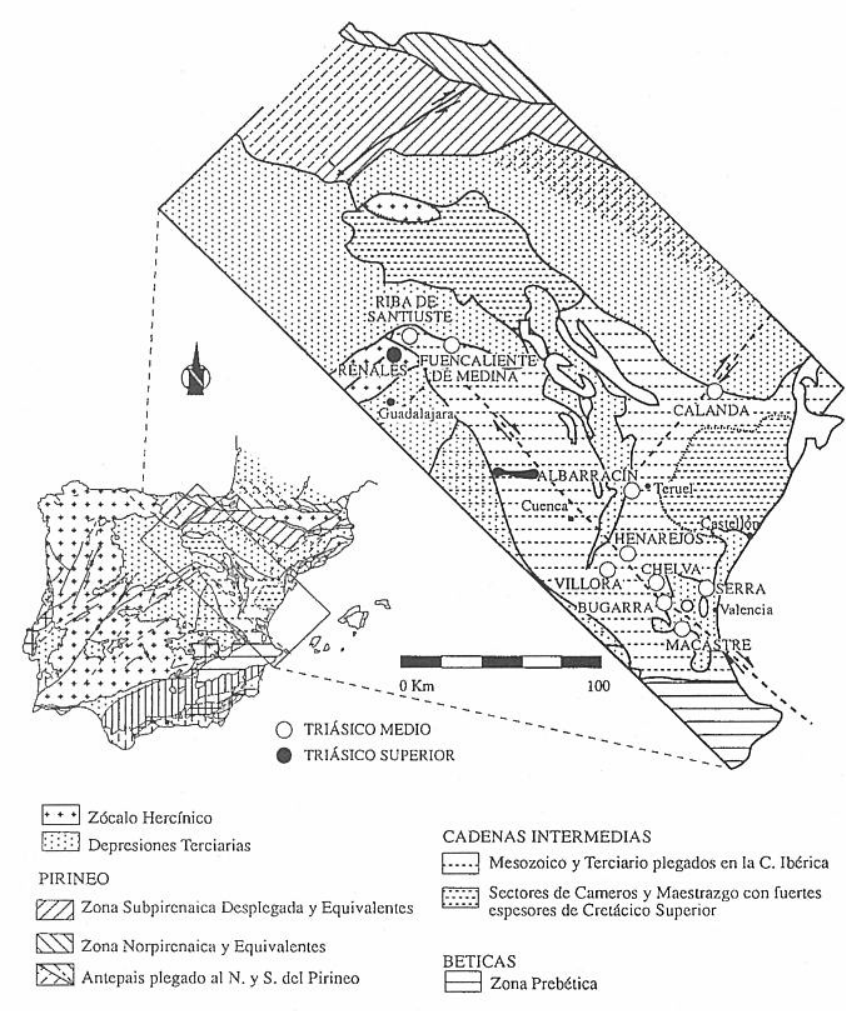

Figura 1. Localización geográfica de los distintos yacimientos fosilíferos estudiados en la Cordillera Ibérica.

\section{Tramo carbonatado inferior del Muschelkalk}

En el Tramo Carbonatado Inferior del Muschelkalk (M1), Formación Dolomías de Landete (López-Gómez y Arche, 
1992; López-Gómez et al, 1993), la presencia de bivalvos determinables es mínima y se han encontrado registros en pocas de las numerosas columnas estudiadas en Teruel, Valencia y Cuenca -Albarracín, Serra, Chelva, Camarena de la Sierra, Ermita y Mas de Herrero (Hinkelbein, 1969; Márquez-Aliaga, 1985; Márquez-Aliaga y López-Gómez, 1989)-. Se ha podido determinar: Hoernesia socialis (Schlotheim), Placunopsis sp., Myophoria vulgaris (Schlotheim), Myophoria cardisoides (Alberti), Myophoria sp., Lyriomyophoria af. elegans (Dunker), Pleuromya elongata (Schlotheim), Pleuromya fassaensis (Wissmann), Neoschizodus laevigatus (Goldfuss), Modiolus sp., Unionites sp., Astartellopsis triasina Roemer y Pseudocorbula sp.. La mayor diversidad específica se ha obtenido en el afloramiento de Serra (MárquezAliaga, 1985) por lo que denominamos a esta asociación "Fauna de Serra" (F-S); está constituida por elementos cosmopolitas del Triásico Medio. El conjunto denota su correspondencia a ambientes marinos someros y/o restringidos.

\section{Tramo carbonatado superior del Muschelkalk}

El Tramo Carbonatado Superior del Muschelkalk (M-3) es el mejor conocido desde la perspectiva paleontológica, y es el que mayor diversidad específica aporta en las áreas estudiadas: Sectores Meridional-Central, Noroccidental y Nororiental.

\section{Sector Meridional-Central.}

Corresponde a las provincias de Teruel, Cuenca y Valencia. En la Formación Dolomías y Calizas de Cañete (LopezGómez y Arche, 1992, 1994; López-Gómez et al, 1993) se han obtenido bivalvos en las columnas de Bugarra, Chelva, Mas de Herrero, Henarejos, Villora, Libros, Villel, Losilla, La Contienda, Moya, Hoz, Huélamo y Barranco de la Hoya (MárquezAliaga, 1985; Márquez-Aliaga et al 1984; López-Gómez et al, 1987; Márquez-Aliaga y López-Gómez, 1989). Entre otras, las especies mejor representadas son: Neoschizodus laevigatus (Goldfuss), Costatoria goldfussi (Alberti), Bakevellia costata (Schlotheim), Bakevellia subcostata (Schlotheim), Leptochondria alberti (Goldfuss), Pseudocorbula gregaria (Munster), Modiolus myoconchaeformis (Philippi), Modiolus salztettensis (Hohenstein), Enantiostreon difforme (Schlotheim), Enantiostreon flabellum (Schmidt), Placunopsis teruelensis Wurm, Gervillia joleaudi (Schmidt). En términos generales, esta asociación coincide para las distintas especies de bivalvos con la denominada por Schmidt (1935) "Fauna de Teruel" que, según el autor, "es muy frecuente en los tramos superiores del Muschelkalk de la Cordillera Ibérica, conocidos como "Capas de Royuela". El material paleontológico procedente de Royuela, área de Albarracín (Teruel), y descrito por Hinkelbein (1969) ha sido revisado por la autora en el "Geol.-Paläont. Institut der Universität" de Stuttgart. La mayor diversidad de esta "Fauna de Teruel" (F-T) coincide con la descrita por Márquez-Aliaga In: Hirsch et al. (1987). Actualmente, las aludidas "Capas de Teruel" se corresponden con la Formacion Dolomías, Margas y Calizas de Royuela (Pérez-Arlucea \& Sopeña, 1985; PérezArlucea, 1992; Pérez-Arlucea \& Rey, 1994).

\section{Sector Noroccidental.}

En la Rama Castellana de la Cordillera Ibérica, provincias de Soria y Guadalajara, el Triásico Medio está constituido por dos formaciones carbonáticas, que se corresponden con el Tramo Carbonatado Superior del Muschelkalk
(M-3): Formación Dolomías de Tramacastilla (D.T.) y Formación Dolomías Margas y Calizas de Royuela (D.M.C.R.) y que hacia el noroeste pasan lateralmente a una formación terrígena, Formación Areniscas y Lutitas de la Cuesta del Castillo, (A.L.C.C.) (García-Gil, 1991, 1994). Los mejores registros proceden de Riba de Santiuste y Fuencaliente de Medina. El contenido paleontológico estudiado por la autora y situado con extraordinario detalle estratigráfico -a nivel milimétrico- por García-Gil (1991), permitió plantear la presencia, en este sector, de tres asociaciones de bivalvos que quedan diferenciadas tanto por su posición estratigráfica como por sus características paleoecológicas (MárquezAliaga y García-Gil, 1991):

1.- La asociación Costatoria-Lyriomyophoria (C-L) se registra en toda la zona, Formaciones A.L.C.C. y D.T., y está constituida por abundantes especímenes de Costatoria goldfussi (Alberti) y Lyriomyophoria sublaevis Schmidt, siendo muy escasa la presencia de Lyriomyophoria af. elegans (Dunker), Neoschizodus laevigatus (Goldfuss), Pleuromya elongata (Schlotheim) y P. brevis (Assman).

2.- La asociación Lingula-Pseudocorbula (Li-P), que se registra en toda la zona pero se corresponde con los niveles estratigráficamente más altos de la formación D.M.C.R., está contituida por la presencia de abundantes especímenes de Pseudocorbula gregaria (Munster) y la presencia escasa del braquiópodo inarticulado "Lingula" (sensu Schmidt, 1935), junto con fragmentos de vertebrados.

3.- La asociación "Fauna de Teruel" (F-T), se registra únicamente en las series más occidentales del área, zona de Fuencaliente de Medina, y aparece intercalada estratigráficamente entre los tramos que contienen las dos asociaciones anteriores. Esta asociación es característica, como ya hemos visto, del Sector Central y Meridional de la Cordillera Ibérica. Su presencia en la provincia de Soria constituye el hallazgo más occidental de la misma y, por ende, es el hallazgo más occidental de bivalvos triásicos cosmopolitas del Tethys.

\section{Sector Nororiental.}

Es la Rama Aragonesa de la Cordillera Ibérica. Los mejores registros proceden del Anticlinal de Calanda, estudiados, inicialmente, por Anadón \& Albert (1973). La colección de estos autores fue revisada por Márquez-Aliaga (1985). Nuevos hallazgos en la zona, tanto de macrofósiles (bivalvos y ammonites), como de microfósiles (conodontos y foraminíferos), permitieron avanzar en el conocimiento de este sector (Márquez-Aliaga et al., 1987). Por último, en Márquez-Aliaga et al. (1994), se integran el contenido fosilífero y el ambiente de sedimentación dẻ la serie de Calanda. Referente a bivalvos, se ha podido determinar: Gervilleia joleaudi (Schmidt), Modiolus myoconchaeformis (Philippi), Pseudocorbula gregaria (Munster), Enantiostreon difforme (Schlotheim), Bakevellia costata (Schlotheim), B. subcostata (Goldfuss), B. crispata (Goldfuss), Entolium discites (Schlotheim), Limea costata (Munster), Myophoria vulgaris (Schlotheim), Neoschizodus laevigatus (Goldfuss) y Pleuromya sp. Las cinco primeras especies se corresponden con elementos característicos de la asociación "Fauna de Teruel" (F-T) junto con otras más representativas del tramo carbonatado superior de las Cordilleras Costero Catalanas. Por ello, y desde una perpectiva paleobiogeográfica, consideramos que podría tratarse de una región relacionada con ambas cuencas. 


\section{Tramo carbonatado del Triásico Superior}

El reciente hallazgo de una asociación de bivalvos en los materiales de la parte superior de la Formación Dolomías Tableadas de Imón (Goy et al., 1976) en una sección del área tipo, Renales (Guadalajara), nos ha permitido avanzar en el conocimiento paleoecológico y bioestratigráfico del Triásico Superior de la Cordillera Ibérica (Goy y Márquez-Aliaga, 1994, en prensa). El registro se corresponde con un conjunto de bivalvos de pequeño tamaño cuya determinación específica permanece imprecisa en algunos casos. La asociación está constituida por: Nuculana cf. borsonii (Stoppani), Rhaetavicula cf. contorta (Portlock), Isognomon cf. exilis (Stoppani), Neoschizodus reziae (Stoppani), Myophoriopis isosceles (Stoppani), Protocardia rhaetica (Merian in Linth) y Laternula cf. amicii (Stoppani).

Entre las especies reconocidas $R$. contorta es característica del "Retiense" (Noriense p.p.) en Europa. Las demás especies han sido citadas como materiales procedentes del Noriense en diferentes localidades alpinas. Por tanto, la Formación Imón representa la transgresión más joven del Triásico de la C. Ibérica.

\section{MENORCA}

Los afloramientos del Muschelkalk de Menorca (Fig. 2) presentan una notable uniformidad litológica, diferenciándose tres tramos que pueden ser identificados en toda la isla. El inferior, de unos $30 \mathrm{~m}$ de potencia (formado por calizas micríticas grises muy bioturbadas), el intermedio, de unos 75 $\mathrm{m}$ de potencia (formado por alternancia de calizas y margas) y el superior, de unos $20 \mathrm{~m}$ de potencia (formado por dolomías). En el tramo intermedio se registran las principales asociaciones de ammonoideos y bivalvos, registrados en las localidades de S'Arenal d'en Castell, El Toro y Sa punta d'es Vernis, y se corresponde con el momento de máxima profundización de la cuenca de los cuatro ciclos de somerización visibles en el tramo (Llompart et al, 1987). Respecto a los bivalvos se ha podido determinar: Daonella lommeli (Wissmann), Posidonia wengensis (Wissmann), Enantiostreon difforme (Schlotheim), Pseudocorbula gregaria (Münster), Cassianella decussata (Münster), Costatoria goldfussi (Alberti) y Astarte cf. triasina (Roemer).

La asociación Daonella-Posidonia (D-P), está constituida por un abundante número de especímenes que se registran en niveles correspondientes a los dos primeros ciclos del tramo. La presencia de $D$. lommeli, especie característica de las capas de Wengen del Triásico alpino (Capoa Bonardi, 1984) indicaría el Ladiniense superior (Longobardiense). La asociación Cassianella-Costatoria (Ca-C) se registra, con abundantes ejemplares, en la parte final del tramo segundo. La presencia de $C$. decussata, especie característica de las capas de S. Cassian del Trias alpino (Schmidt, 1935) indicaría el Carniense. El Muschelkalk de Menorca ofrece los mejores registros de ammonites del Triásico hispano. Ello, en correlación con las asociaciones de bivalvos citadas, permite verificar su pertenencia a las Zonas Archelaus (D-P) y Aon (Ca-C), respectivamente.

\section{CORDILLERA COSTERO CATALANA}

Las secuencias carbonatadas de la Cordillera Costero Catalana (Fig. 2), al igual que en los sectores orientales de la Cordillera Ibérica y de la Depresión del Ebro, están representadas por el Muschelkalk inferior, Muschelkalk superior y Formación Imón, de edades Anisiense, Ladiniense y Retiense respectivamente (Calvet et al., 1994c).

\section{Muschelkalk Inferior}

El Muschelkalk Inferior, estudiado desde la perspectiva estratigráfica y sedimentológica con gran detalle por Calvet et al. (1990) y Calvet y Marzo (1994), tiene una potencia que oscila entre 70-120 m en el sector occidental. Ha sido subdividido en cuatro unidades que, de muro a techo, son: Unidad Brull, U. Olesa, U. Vilella Baixa y U. Colldejou. El hecho de que las mismas se encuentran total o parcialmente dolomitizadas podría justificar, desde la perpectiva tafonómica, el bajo registro de fósiles carbonáticos y más concretamente de macrofósiles determinables.

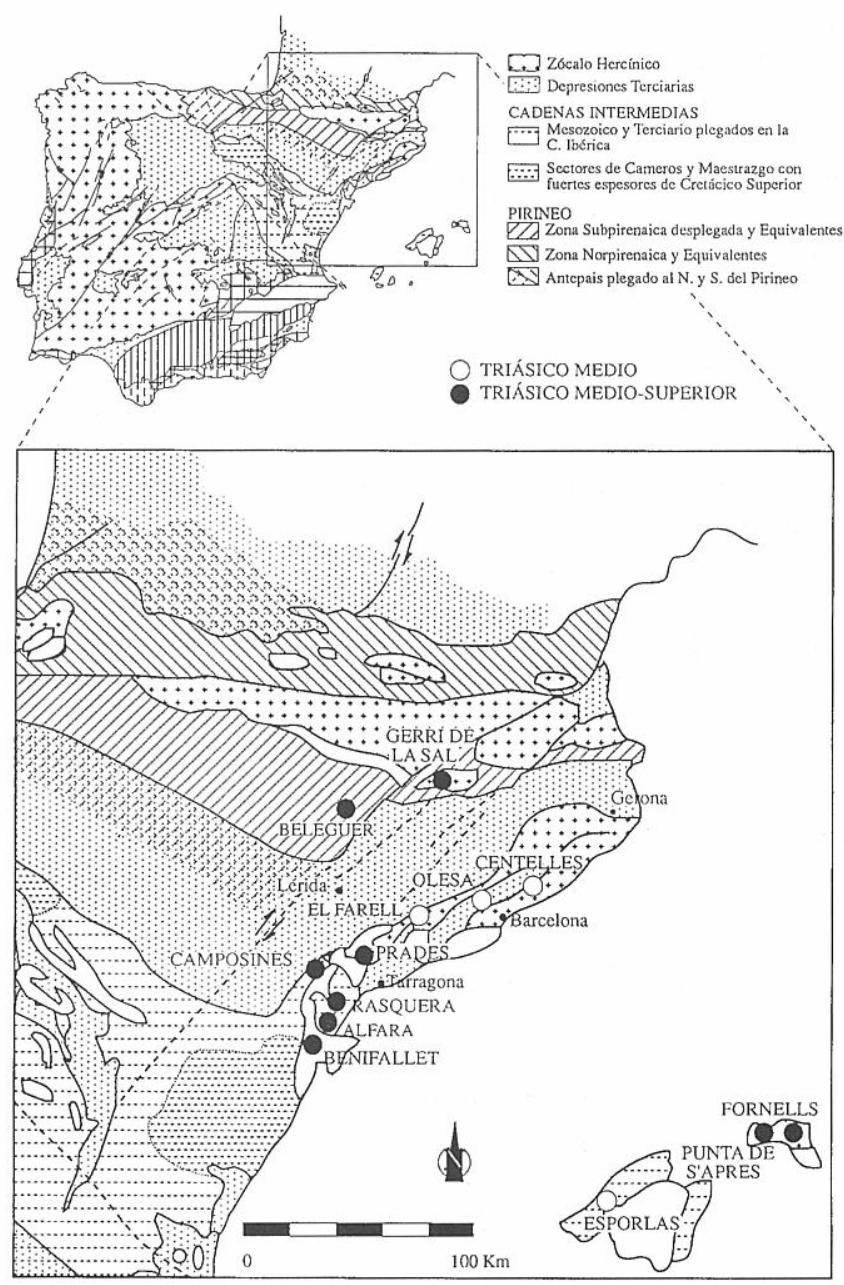

Figura 2. Localización geográfica de los distintos yacimientos, fosilíferos estudiados en las Cordilleras Costero Catalana, Pirineos Catalanes y Menorca. 
En lo referente a los bivalvos ${ }^{1}$, los ejemplares mejor estudiados proceden de las columnas de Olesa, Centelles y El Farell (Márquez-Aliaga, 1985), siendo representativas las siguientes especies: Entolium discites (Schlotheim), Paleonucula goldfussi (Alberti), Parallelodon beyrichy (Strombeck), "Mytilus" eduliformis (Schlotheim) y Pleuronectites laevigatus (Schlotheim), Plagiostoma cf. striata (Schlotheim), Pachymia sp., Chlamys sp., Claraia sp., Pleuromya brevis (Assmann); junto con registros que hemos caracterizado como representantes de la asociación "Fauna de Serra" (FS) del tramo carbonático equivalente en la Cordillera Ibérica. El conjunto ofrece las mismas consideraciones que las antedichas para la citada asociación.

\section{Muschelkalk Superior}

El Muschelkalk Superior, cuya potencia varía entre 100$140 \mathrm{~m}$ en las áreas más septentrionales, ha sido dividido en cinco unidades que, de muro a techo, son: Rojals, Benifallet, Rasquera, Tivisa y Capafons. Son distintas segun el Dominio estudiado: Gaiá-Montseny, Prades y Baix Ebre-Priorat, de NE a SW.

La unidad de la Pedra d'Alcover rellena las depresiones de los complejos arrecifales del Dominio de Prades, siendo concordante con la U. Benifallet en las áreas interarrecifales. Desde la perspectiva paleontológica, merece mención aparte por su alto contenido en fósiles; están bien representados artrópodos, peces, celentereos, moluscos y plantas. Este yacimiento fue considerado por Via y Villalta (1977) como equivalente, desde la perspectiva tafonómica, al de Solenhofen en el Jurásico de Baviera; se trata de un verdadero "Lagerstatten" para el Triásico español. Respecto a los bivalvos, estudiados por la autora tanto en el Museo del Seminario de Barcelona como en el Museo de Alcover, verificamos la presencia de las especies: Daonella lommeli (Wissmann), Entolium discites (Schlotheim), Enantiostreon difforme (Schlotheim), Bakevellia costata (Schlotheim), Modiolus sp. y Pseudocorbula sp.

La unidad de Rasquera se localiza en el Dominio del Baix Ebre-Priorat. La potencia, máxima de $40 \mathrm{~m}$ en el Baix Ebre, disminuye hasta unos pocos metros en el Dominio del Priorat. La Unidad está constituida por cinco ciclos formados, a su vez, por cinco facies diferentes (Calvet y Tucker, 1988). La segunda facies está formada por alternancia de niveles de Lutitas y de Calizas de tamaño centimétrico. Tanto en la base como en el techo de los niveles calcáreos es frecuente el registro de Daonella en disposición horizontal. En las localidades de Camposines, Alfara y Begues hemos encontrado abundantes ejemplares de Daonella lommeli (Wissmann) y Posidonia wengensis (Wissmann) (asociación D-P) (Márquez-Aliaga, 1985). Su presencia confirma la interpretación de esta facies

1 Nuestro estudio se ha basado, fundamentalmente, en la colección paleontológica utilizada por Virgili (1958) y estudiada por el Dr. Villalta. La citada colección (depositada en el Dpto. de Geología de la Universidad de Valencia) fue revisada por la autora (Márquez-Aliaga, 1985). La misma constituye un conjunto de extraordinario valor paleontológico pero, en la mayoría de los casos, no se conoce con detalle el nivel de procedencia de algunos bivalvos, por ello nos referimos a los datos obtenidos, tras la revisión taxonómica efectuada, y de manera general respecto a los tramos carbonáticos inferior y superior de Muschelkalk sin detallar su ubicación en las unidades descritas posteriormente por Calvet (op. cit.). como perteneciente a un ambiente de rampa profunda distal y permite atribuir una edad Ladiniense superior (Longobardiense) a los niveles que registran la citada asociación; ello está de acuerdo con los datos aportados por los conodontos (March, 1991) y los foraminíferos (Márquez, 1994). A techo de los ciclos de la unidad Rasquera, se presenta una facies (la quinta) constituida por "Packstones-lumaquela" y en ella se registran bivalvos y ammonites. Entre los bivalvos se determinan las especies: Entolium discites (Schlotheim), Limea vilasecai (Schmidt), Limea costata (Goldfuss), Bakevellia subcostata (Goldfuss), Pleuromya elongata (Schlotheim), Chlamys sp.. Por otro lado, se registran especies que, en parte, se incluyen entre las que hemos definido para la Cordillera Ibérica como pertenecientes a la asociación Fauna de Teruel (F-T). Su presencia implicaría la pertenencia de esta facies, final de cada ciclo, a un ambiente proximal y/o submareal. Los bivalvos mejor conservados proceden de la localidad de Benifallet (a techo del tercer ciclo de la unidad). En conjunto, se han determinado las especies: Entolium discites (Schlotheim), Limea vilasecai (Schmidt), L. costata (Goldfuss), Bakevellia subcostata (Goldfuss), Pleuromya elongata (Schlotheim), Chlamys sp., Lyriomyophoria sp. y, con menor representación, algunas especies comunes a las que hemos incluido en la asociación "Fauna de Teruel" (F-T).

Virgili (1958) cita como procedentes de los niveles terminales del Muschelkalk (M-3), en tramos equivalentes al "Lettenkohle" alemán, de Alfara, La Musara, Camposines y Mas Fonoll de Pontos, las especies: Costatoria goldfussi (Alberti), Lyriomyophoria sublaevis Schmidt y Cassianela decussata (Munster). Denominamos a este conjunto asociación Casianella-Costatoria (Ca-C). Por la procedencia citada, esta asociación podría situarse a techo de la unidad Capafons -equivalente a la Formación Royuela de la Cordillera Ibérica (Calvet \& Marzo, 1994)- y se corresponde con ambientes poco profundos en medios altamente inestables y restringidos. Podría indicar una edad Ladiniense superiorCarniense; C. decussata es abundante en las Capas de St. Cassian en el Carniense de los Alpes.

\section{Formación Imón}

Se localiza en las proximidades de Alfara de Carlet, presenta los miembros característicos de esta Formación (Calvet y Marzo, 1994) y un nivel con registro de bivalvos que se están estudiando (Márquez-Aliaga y Calvet, en preparación).

\section{PIRINEOS CATALANES}

El Triásico de la zona de Gerri de la Sal (Les Nogueres) (Fig. 2) presenta los cuatro tramos característicos del Triásico de los Pirineos, que de base a techo son: Buntsandstein, Muschelkalk, Keuper y Formación Isábena. El Muschelkalk está constituido por tres unidades litoestratigráficas. La tercera, constituida por calizas y/o dolomías tableadas, de 15 a $30 \mathrm{~m}$ de potencia contiene niveles centimétricos de "Packstones" de bivalvos (Calvet et al, 1994b). Se ha podido determinar: Myophoriopis keuperina (Quensted), Unionites muensteri (Wissmann), Pseudocorbula gregaria (Munster), Pleuromya elongata (Schlotheim), Neoschizodus laevigatus (Goldfuss) y Bakevellia sp. 
El registro de ejemplares de muy pequeña talla que muestran sus dos valvas unidas y abiertas, nos indica la existencia de medios anóxicos y sin energía hidráulica. La talla pequeña podría corresponder a intentos de colonización, rápidamente abortados, en medios altamente inestables. $M$. keuperina y $U$. Münsteri (=Anoplophora) se citan frecuentemente en el "Lettenkhole" alemán. En conjunto, la asociación no sería incompatible con el Ladiniense superior-Carniense.

En el Triásico de las Sierras Marginales de la zona de Baleguer (Lerida), y en tramos equivalentes a los anteriormente citados en la zona de Gerri de la Sal, se ha podido determinar Pseudocorbula gregaria (Munster), Pseudocorbula sp. y Bakevellia sp. La presencia del erizo Cidarites cf. grandaevus (Alberti), registrado en un nivel con acumulación de puas de tamaño pequeño, que es característico del Trochitenkalk ("Muschelkalk superior") de Crailsheim (Alemania) (Hagdorn, 1988) nos permite atribuir el conjunto al Ladiniense.

\section{CORDILLERA BÉTICA}

La Cordillera Bética (Fig. 3) puede ser dividida en dos dominios, las Zonas Externas y las Zonas Internas, con diferente significado estructural. Las Zonas Externas comprenden: la Zona Prebética, que permaneció en una posición próxima a la Meseta durante el depósito de sedimentos continentales y marino-someros del Mesozoico, y la Zona Subbética situada al sur. Las facies triásicas que aparecen en estos dominios son de tipo Germánico equivalentes a las que se encuentran en las Cordilleras Ibérica, Catalana y Cantá-

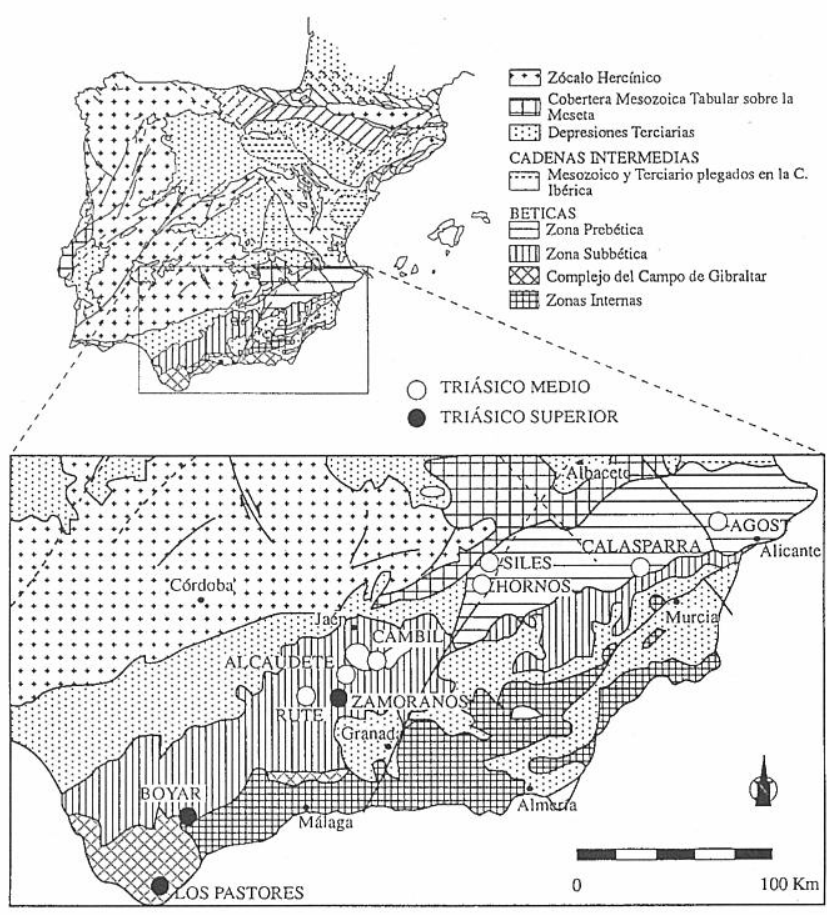

Figura 3. Localización geográfica de los distintos yacimientos fosilíferos estudiados en las Cordilleras Béticas (Zonas externas: Prebético y Subbético). brica (Martín-Algarra et al., 1994; Fernández et al, 1994; Pérez-López \& Sanz de Galdeano, 1994). Las Zonas Internas están compuestas por tres complejos estructurales tectónicamente superpuestos, denominados del más bajo al más alto: Nevado Filábride, Alpujárride y Maláguide; los dos primeros presentan un triásico de facies alpina , pero el último incluye facies pericontinentales ("facies Verrucano") del Triásico.

En este trabajo se trata el conocimiento actual de los bivalvos en tres sectores de las Zonas Externas de la Cordillera Bética: 1. Oriental (Prebético), 2. Central (Subbético), 3. Occidental (Subbético).

\section{CORDILLERA BÉTICA ORIENTAL}

\section{Área de Alicante}

A principios de siglo, el Triásico de Alicante fue objeto de numerosos estudios paleontológicos. Los yacimientos de Font de la Reina, Espejeras y Agost, entre otros, fueron atribuidos por primera vez al "Raibliense" por Jiménez de Cisneros (1929). Posteriormente, Schmidt (1935) describe en los mismos veinticinco especies de bivalvos, de los cuales nueve son taxones nuevos. La discusión sistemática sobre estas especies fue llevada a cabo por Márquez-Aliaga (1985). Márquez-Aliaga y Montoya (1991) revisan las colecciones depositadas en el "Museu Històric Municipal" de Novelda, con bivalvos de extraordinaria preservación, aunque sin ubicación estratigráfica precisa. El estudio estratigráfico, sedimentológico y paleontológico de las secciones del Muschelkalk de los alrededores de Agost (columna de Espejeras) es muy reciente (López-Gómez et al., 1994). El Muschelkalk de Espejeras tiene $145 \mathrm{~m}$ de potencia, siendo los últimos $50 \mathrm{~m}$ los que registran frecuentes macrofósiles. Este estudio nos ha permitido situar los bivalvos (encontrados por nosotros y parte de los revisados en Museos) en su contexto bioestratigráfico y relacionarlos con los foraminíferos y los cefalópodos registrados. Consideramos que la asociación F-T está bien representada en todo el tramo, pero en los niveles terminales es mayoritaria la presencia de Costatoria goldfussi y minoritaria la de Lyriomyophoria sublaevis (C-Ly).

El registro, en la parte superior de Espejeras y Font de la Reina, de: cefalópodos -Germanonautilus bidorsatus (Schlotheim), y Ceratites $c f$. nodosus (Schlotheim) colonizados con el bivalvo Placunopsis ostracina (Schlotheim) (Márquez-Aliaga y Martínez, 1994; Goy y Martínez, 1994)-, Casianella decussata (Münster) (Schmidt, 1935), y el reciente hallazgo de Pseudofurnishius murcianus (Boogaard) (Martínez et al., 1994, en prensa), permite caracterizar el Ladiniense Superior-Carniense.

\section{Área de Murcia}

El tramo cabonatado del Muschelkalk en el área de Murcia alcanza una potencia próxima a los $150 \mathrm{~m}$. En los niveles superiores del tramo (localidades de Cehegin, Caravaca, Calatrava y Mula) la presencia de placas con abundantes bivalvos es frecuente. Se aporta, por primera vez, el resultado del estudio de los bivalvos de esta zona, que forma parte de un trabajo estratigráfico y paleontológico inédito (Calvet 
y Márquez-Aliaga, en preparación). El Muschelkalk de Cehegin, al igual que el de Espejeras (Márquez-Aliaga y Montoya, 1991), estudiado paleontológicamente por Schmidt (1935) sirvió de base para la creación de algunas especies nuevas para el Triásico español: Avicula hallensis Wohrman (=Bakevellia gansingensis, Alberti), Myophoria laevigata Alberti (= Neoschizodus laevigatus Goldfuss), M. kiliani sp.nov. (=Costatoria goldfussi Alberti), M. sublaevis sp. nov. (=Lyriomyophoria sublaevis Schmidt) y Mytilus eduliformis Schlotheim (="Mytilus" eduliformis) (entre paréntesis nuestras sinonimias). Por otro lado, hemos podido determinar: Placunopsis cf. teruelensis Wurm, P. ostracina (Schlotheim), Bakevellia costata (Schlotheim), Enantiostreon difforme (Schlotheim), Modiolus myoconchaeformis (Philippi), Entolium cf. discites (Schlotheim) y Chlamys sp.

La abundancia de $C$. goldfussi y L. sublaevis es manifiesta, y por tanto, está bien representada la asociacion (C-L). $\mathrm{El}$ resto de las especies contituyen algunos elementos característicos de la asociacion (F-T). La presencia de Bakevellia gansingensis, aunque escasa, es característica del tránsito al Triásico Superior. Por tanto, al igual que en el área de Alicante, las especies de bivalvos estudiadas constituyen asociaciones del Ladiniense Superior-Carniense en idénticos ambientes sedimentarios que los descritos en Espejeras por López-Gómez et al. (1994).

\section{CORDILLERA BÉTICA CENTRAL}

\section{Área de Jaén}

Los materiales carbonatados de facies Muschelkalk en la Zona Prebética, al norte de Jaén, están constituidos por bancos dolomíticos separados por lutitas con algunas evaporitas y niveles arenosos. Las tres intercalaciones carbonáticas de 8,20 y $25 \mathrm{~m}$ de potencia, constituyen la Formación Hornos-Siles, de $385 \mathrm{~m}$ de potencia, formada, mayoritariamente, por materiales detríticos y evaporíticos. Los bivalvos han sido estudiados por Márquez-Aliaga et al. (1986) sobre un conjunto, superior a mil especímenes, procedentes de Orcera, Cortijos Nuevos, Hornos y Siles; (incluyendo la colección de ejemplares de Hornos-Siles depositada en el "Museum d'Histoire Naturelle" de Ginebra, revisados por Márquez-Aliaga). Se han podido determinar las especies: Modiolus cf. myoconchaeformis (Philippi), Bakevellia costata (Schlotheim), Gervillia joleaudi (Schmidt), Leptochondria alberti (Goldfuss), Enantiostreon flabellum (Schmidt), Placunopsis teruelensis Wurm, Costatoria goldfussi (Alberti), Neoschizodus cf. laevigatus (Goldfuss), Pseudocorbula gregaria (Münster) y Pleuromya cf. elongata (Schlotheim).

En el primer tramo carbonatado es dominante la presencia de E. flabellum. Hacemos constar que se trata de una especie nueva para el Triásico español y que la dificultad de interpretación de estas "falsas ostras" es grande. Su proximidad a otras especies descritas en el Anisiense del Valle del Jordan (Márquez-Aliaga et al., 1986) nos aconseja la revisión de este taxón (Márquez-Aliaga y Seilacher, en preparación). En el tercer tramo se registran la mayoría de las especies citadas, que asimilamos a la asociación F-T; pero es mayoritaria la presencia de $P$. gregaria y $C$. costata en los niveles termina- les del mismo. Otras evidencias bioestratigráficas, con polen, ammonites y conodontos, permiten atribuir una edad Ladiniense superior a los últimos tramos de la citada Formación.

\section{Área de Córdoba}

Los materiales triásicos situados al sur de la depresión del Guadalquivir, entre Puente Genil (Córdoba) y Cabra de Santo Cristo (Jaén), han sido estudiados por Pérez-López (1991). En la zona se diferencian dos tramos carbonatados: uno inferior de facies Muschelkalk (Formación Majanillos) del Ladiniense, y otro superior (Formación Zamoranos) del Noriense.

\section{Formación Majanillos}

Perez-López (1991) define la Formación Majanillos para los carbonatos de facies Muschelkalk de la Zona Subbética. $\mathrm{Su}$ potencia oscila entre 80-130 m y está limitada, a muro y techo, por materiales detríticos a través de contactos mecánicos. Los bivalvos se registran siempre en los niveles más altos de las series pero están mal conservados. Los mejores hallazgos se sitúan en las localidades de Jauja, Alcaudete, Valdepeñas, Cambil y Cabra (Márquez-Aliaga, In: Pérez-López, 1991 y Pérez-López et al., 1991). Se han determinado las especies: Bakevellia costata (Schlotheim), Enantiostreon cf. flabellum (Schmidt), E. difforme (Schlotheim), Placunopsis teruelensis Wurm, Costatoria goldfussi (Alberti), Neoschizodus cf. laevigatus (Goldfuss), Pseudocorbula gregaria (Münster), Limea costata (Goldfuss), Unionites sp., y Modiolus sp.

Este tipo de asociación es muy parecida a la que se registra en el área de Hornos-Siles pero con abundante Costatoria y Pseudocorbula; estas especies son características de un ambiente inter-supralitoral en medios inestables y condiciones regresivas. Se trata de fósiles de facies cuyas especies tienen amplios rangos bioestratigráficos. Sin embargo, el conjunto puede considerarse como característico del Ladiniense en la parte occidental del Dominio Sefardí (MárquezAliaga \& Hirsch, 1988; Hirsch \& Márquez-Aliaga, 1988).

\section{Formación Zamoranos}

La Formación Zamoranos está formada por un tramo carbonático cuya potencia no supera los $40 \mathrm{~m}$. Algunos autores la consideraron del Muschelkalk; recientemente, PérezLópez (1991) planteó su posible correlación con la Formación Imón de la Cordillera Ibérica. Su atribución cronoestratigráfica al Noriense medio-superior (Pérez-López et al., 1992) se basa en la presencia de polen, coprolitos, foraminíferos y bivalvos: Chlamys cf. valoniensis (Defrance), Chlamys sp. y Entolium sp.

\section{CORDILLERA BÉTICA OCCIDENTAL}

\section{Área de Cádiz-Algeciras}

En el Trias Superior de Los Pastores (Cádiz), en los alrededores de Algeciras, Martin-Algarra et al. (1993, 1994) diferencian siete Unidades. Las cuatro inferiores de tipo Keuper, en donde se ha registrado polen del Carniense, y las tres últimas de naturaleza carbonatada, que se corresponden con 
ambientes marinos de plataforma somera e influencia mareal con las siguientes especies: Lyriomyophoria sublaevis Schmidt, Pseudocorbula gregaria (Münster), Enantiostreon cf. flabellum Schmidt, Gervillia joleaudi Schmidt y Gervillia subcostata (Goldfuss). La presencia de L. sublaevis es mayoritaria y está bien representada $G$. costata. Esta última ha sido citada en niveles del "Grenzdolomit" alemán de edad Carniense.

\section{Área de Cádiz-Boyar}

El Corredor del Boyar es un área deprimida entre las localidades de Ubrique y Grazalema, en la provincia de Cádiz. En el Puerto de Boyar se pueden diferenciar dos unidades litológicas mayores: Una inferior carbonatada, con 70 $\mathrm{m}$ de potencia de facies Muschelkalk, en donde se registra polen, bivalvos, braquiópodos, gasterópodos y nautiloideos. Otra superior arcilloso-areniscoso-evaporítica, de facies Keuper. Ambas han proporcionado microflora de edad Carniense superior (Martín-Algarra et al., 1994).

Se han reconocido dos conjuntos diferentes de bivalvos. El primero se registra en la parte media de la unidad carbonatada, formado por: Placunopsis ostracina (Schlotheim), P. plana (Giebel), Enantiostreon sp., Costatoria goldfussi (Alberti), Entolium cf. subdemissum (Münster), Entolium sp., Gervillia joleaudi (Schmidt), Septihoernesia cf. joannisaustriae (Klipstein), Bakevellia gassingensis (Alberti), Bakevellia sp., Chlamys cf. subequicostatus (Bittner), Chlamys sp.. El segundo se registra en un único nivel carbonatado, que se sitúa en la zona de transición a la unidad superior de facies Keuper; está formado por: Paleonucula cf. strigilata (Goldfuss), Pseudocorbula gregaria (Münster), P. cf. subundata (Schauroth), Unionites muensteri (Wissmann), Modiolus sp., Placunopsis ostracina (Schlotheim), P. Plana (Giebel) y Bakevellia sp.

Desde el punto de vista bioestratigráfico, las especies Entolium cf. subdemissum, Septihoernesia cf. joannisaustriae, Bakevellia gassingensis, Paleonucula cf. strigilata, Pseudocorbula cf. subundata han sido frecuentemente citadas en el Carniense de Lombardía. El resto lo constituyen especies de amplio rango que ya hemos citado en niveles carbonáticos del Triásico Medio.

\section{CONCLUSIONES}

Los resultados obtenidos permiten definir varias asociaciones de bivalvos que resultan características de las distintas Formaciones del Triásico Medio de las Cordilleras Costero Catalanas, Cordillera Ibérica, Menorca y del Sector Central de las Zonas Externas de las Cordilleras Béticas. Las asociaciones del Triásico Superior se están estudiando en la actualidad, pero contamos con algunos datos puntuales de la Cordillera Ibérica, de las Zonas Externas de la Cordillera Bética (Prebético y Subbetico) y de Menorca.

Diferenciamos siete asociaciones de bivalvos que consideramos representativas de los tramos carbonatados del Triásico español en los distintos dominios estudiados. Existen otros registros del Triásico superior los cuales contituyen, posiblemente, otras dos asociaciones que, de momento, no se precisan. Las asociaciones definidas estan constituidas por una serie de especies caracteristicas y se denominan:
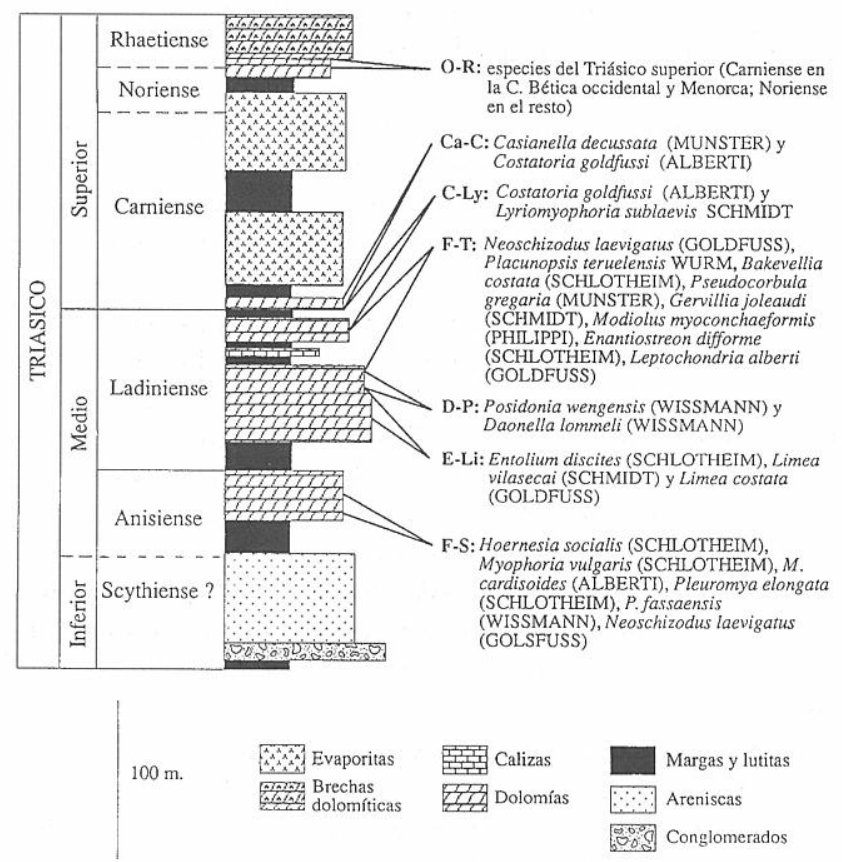

Figura 4. Columna sintética del Triásico Medio y Superior de la Cordillera Ibérica (Sector Meridional) y Costero Catalanas. Se sitúan, en los tres tramos carbonatados, la distribución bioestratigráfica, aproximada, de las distintas asociaciones de bivalvos y sus especies más representativas.

1. Fauna de Serra (F-S): Hoernesia socialis (Schlotheim), Myophoria vulgaris (Schlotheim), Pleuromya elongata (Schlotheim), Pleuromya fassaensis (Wissman) y Neoschizodus laevigatus (Goldfuss).

2. Fauna de Teruel (F-T): Neoschizodus laevigatus (Goldfuss), Placunopsis teruelensis Wurm, Bakevellia costata (Schlotheim), Pseudocorbula gregaria (Münster), Gervi-

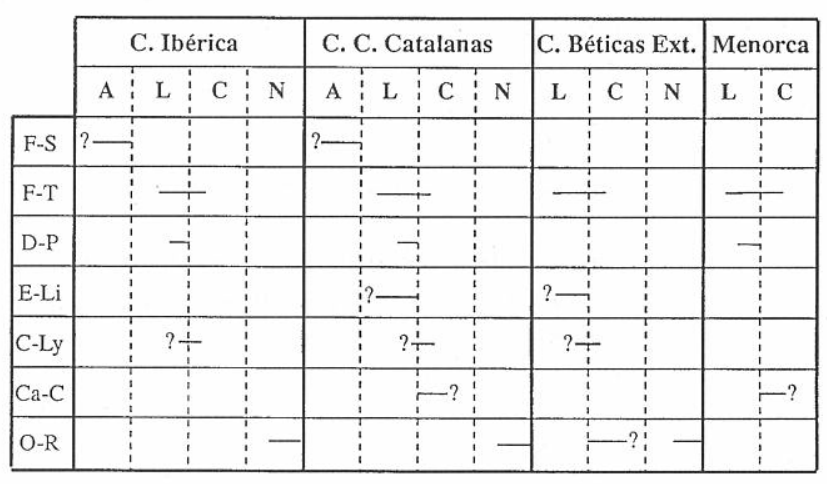

Tabla 1. Distribución bioestratigráfica de las distintas asociaciones de bivalvos triásicos españoles registradas en los distintos tramos carbonatados del Triásico Medio y Superior de las Cordilleras Ibérica, Costero Catalana, Béticas (Zonas Externas) y Menorca. A: Anisiense; L: Ladiniense; C: Carniense; N: Noriense. Asociaciones: F-S: Fauna de Serra; F-T: Fauna de Teruel; D-P: Daonella-Posidonia; E-Li: Entolium-Limea; C-Ly: Costatoria-Lyriomyophoria; Ca-C: Cassianella-Costatoria; O-R: Otros registros del Triásico superior. 
llia joleaudi (Schmidt), Modiolus myoconchaeformis (Philippi), Enantiostreon difforme (Schlotheim), Leptochondria alberti (Goldfuss).

3. Daonella-Posidonia (D-P): Posidonia wengensis (Wissmann) y Daonella lommeli (Wissmann).

4. Entolium-Limea (E-L): Entolium discites (Schlotheim), Limea vilasecai (Schmidt) y Limea costata (Goldfuss).

5. Costatoria-Lyriomyophoria (C-Ly): Costatoria goldfussi (Alberti) y Lyriomyophoria sublaevis Schmidt.

6. Cassianella-Costatoria (Ca-C): Casianella decussata (Münster) y Costatoria goldfussi (Alberti).

7. Otros Registros $(\boldsymbol{O}-\boldsymbol{R})$ : se agrupan especies del Triásico Superior de hallazgos recientes y distribución puntual.

El registro fósil se corresponde con ambientes marinos protegidos y/o de rampas carbonatadas someras. La mayoría de los taxa son cosmopolitas y comunes en la cuenca del Tethys.

Las especies de bivalvos no tienen significado bioestratigráfico preciso, dado su amplio rango. Sin embargo, sus asociaciones permiten caracterizar las diferentes intercalaciones carbonatadas de la Península Ibérica, tal como queda sintetizado en la Tabla 1 y en la Figura 4.

\section{AGRADECIMIENTOS}

Son muchas las personas cuya contribución ha permitido la elaboración de esta síntesis. Agradecemos, entre otros, a los Profesores Dres.: A. Arche, A. Goy, J. López-Gómez, C. Virgili (U. C. Madrid); F. Calvet (U. Barcelona); M. De Renzi, L. Márquez (U. Valencia); A. Martín-Algarra, A. Pérez-López (U. Granada); S. García-Gil (U. Vigo); F. Hirsch (S. G. Israel); M. Entcheva (U. Sofia); K. Budurov (S. A. Bulgaria); H. Hagdorn (H. M. Museum, Ingelfingen); A. Seilacher (U. Tübingen). A los correctores cuya crítica ha mejorado el trabajo y a nuestro compañero M. Pardo por su ayuda en la informatización de las figuras. Trabajo subvencionado por los proyectos PB91-0644, PB92-0041 (DGICYT) y 075-IVEI.

\section{BIBLIOGRAFÍA}

Anadón, P. y Albert, J. F. 1973. Hallazgo de una fauna del Muschelkalk en el Trias del anticlinal de Calanda. Acta Geológica Hispánica, 5, 151-152.

Bataller, J. R. 1954. Enumeración de las especies nuevas del Triásico y Jurásico de España. Estudios Geológicos, 10 (21), 49-77.

Budurov, K., Calvet, F., Goy, A., Márquez-Aliaga, A., Márquez, L., Trifonova, E. and Arche, A. 1993. Middle Triassic Stratigraphy and Correlation in Part of the Tethys Realm (Bulgaria and Spain). In: Muschelkalk Schöntaler Symposium 1991 (Eds. H. Hagdorn \& A. Seilacher). Ed. Goldschneck, Stuttgart. 157-164. Sonderbände der Gesellschaft für Naturkunde in Würtemberg 2. 288 pp.

Calvet, F., Berástegui, X., Florensa, R. M., Losantos, M., Puig, C., Márquez, L., Márquez-Aliaga, A., Solé de Porta, N. y Trifonova, E. 1994a. El Triásico (Muschelkalk) de la Zona de Guerri de la sal (Les Nogueres, Pirineos Catalanes). In: Resumenes de las Comunicaciones, III Coloquio de Estratigrafía y Paleogeografía del Permico y Triásico de España. (Ed. A. Arche). Cuenca, 27-28.

Calvet, F., Márquez, L. y Trifonova, Ek. 1994b. Litoestratigrafía y bioestratigrafía (Foraminíferos) de facies Muschelkak del Triásico sudpirenaico y del Pirineo Vasco-Cantábrico. Boletín de la Real Sociedad Española de Historia Natural (Sección Geología), 89 (1-4), 167-188.

Calvet, F. y Marzo, M. 1994. El Triásico de las Cordilleras Costero Catalanas: estratigrafía, sedimentología y análisis secuencial. Guia de Campo, III Coloquio de Estratigrafía y Paleogeografía del Pérmico y Triásico de España. (Ed. A. Arche). Cuenca. $53 \mathrm{pp}$.

Calvet, F. and Tucker, M. E. 1988. Outer ramp cycles in the Upper Muschelkalk of the Catalan Basin, northeast Spain. Sedimentary Geology, 57, 185-198.

Calvet, F., Tucker, M. E. and Henton, J. M. 1990. Middle Triassic carbonate ramp systems in the Catalan Basin, northeast Spain: facies, systems tracts, sequences and controls. In: Carbonate platforms: Facies, Sequences and Evolution (Eds. M. E. Tucker, J. L. Wilson, P. D. Crevello, J. R. Sarg \& J. F. Read). Special Publication int. Ass. Sedimentol., 9, 79-108.

Calvet, F., Tucker, M. and Pomar, L. 1994c. Análisis secuencial y ciclicidad de alta frecuencia en el Triásico del nordeste de la Península Ibérica. In: Resumenes de las Comunicaciones, III Coloquio de Estratigrafía y Paleogeografía del Permico y Triásico de España. (Ed. A. Arche). Cuenca, 29-30.

Capoa Bonardi, P. de 1984. Le Daonelle e le Halobie della serie calcareo-silico-marnosa della Lucania (AppeninoMeridionale). Studio paleontologico e biostratigrafico. Memorie della Societá Naturale in Napoli, 78, 1-130.

Cortázar, D. 1875. Descripción física, geológica y agrológica de la provincia de Cuenca. Memorias del Instituto Geológico y Minero de España, 3 (16), 1-406.

Cortázar, D. 1885. Bosquejo físico-geológico y minero de la provincia de Teruel. Boletín de la Comisión del Mapa Geológico de España, 12, 263-607.

Darder, B. 1914. El Triásico de Mallorca. Trabajos del Museo Nacional de Ciencias Naturales (Serie Geológica), 7,1-85.

Dereims, A. 1898. Recherches géologiques dans le Sud de l'Aragón. Thèse Universitaire, Paris, 198 pp.

Fernández, J., Dabrio, C. J. y Pérez-López, A. 1994. El Triásico de la región de Siles Alcaraz (Cordillera Bética). Guia de Campo, III Coloquio de Estratigrafía y Paleogeografía del Permico y Triásico de España. (Ed. A. Arche). Cuenca. 47 pp.

García-Gil, S. 1991. Las unidades litoestratigráficas del Muschelkalk en el NW de la Cordillera Ibérica (España). Boletín de la Real Sociedad Española de Historia Natural, 86 (1-4), 21-51.

García-Gil, S. 1994. El Triásico de la región de Riba de SantiusteArcos de Jalón. Guia de Campo, III Coloquio de Estratigrafía y Paleogeografía del Permico y Triásico de España. (Ed. A. Arche). Cuenca. $52 \mathrm{pp}$.

Goy, A. 1986. Ammonoideos del Triásico de España (Resumen). Universidad Complutense de Madrid (inédita), 28 pp.

Goy, A. (1995). Ammonoideos del Triásico Medio de España: Bioestratigrafía y Correlaciones. Cuadernos de Geología Ibérica, 19; 21-61.

Goy, A., Gómez, J. J. y Yébenes, A. 1976. El Jurásico de la Rama Castellana de la Cordillera Ibérica (Mitad Norte). I. Unidades litoestratigráficas. Estudios Geológicos, 32, 391-423. 
Goy, A. y Márquez-Aliaga, A. 1994. Bivalvos Norienses de la Formación Imón en Renales (Guadalajara, España). In: Resúmenes de las Comunicaciones, III Coloquio de Estratigrafía y Paleogeografía del Pérmico y Triásico de España, 47-48.

Goy, A. y Márquez-Aliaga, A. (En prensa). Bivalvos del Triásico Superior en la Formación Imón (Cordillera Ibérica, España). Boletín de la Real Sociedad Española de Historia Natural (Sección Geología).

Goy, A. y Martínez, G. 1994. Nautiloideos del Triásico Medio en la Cordillera Ibérica y en la parte oriental de las Cordilleras Béticas. In: Resúmenes de las Comunicaciones, III Coloquio de Estratigrafía y Paleogeografía del Pérmico y Triásico de España, (Ed. Arche). Cuenca, 49-50.

Hagdorn, H. 1988. Neue Seeigelfunde aus dem Trochitenkalk von Crailsheim. In: Sonderbände der Gessellschaft für Naturkunde in Wïttemberg, Band 1. (Ed. H. Hagdorn). Stuttgart, 105-115.

Hinkelbein, K. 1969. El Triásico y el Jurásico de los alrededores de Albarracín, Instituto de Estudios Turolenses, 41, 35-75.

Hirsch, F. and Márquez-Aliaga, A. 1988. Triassic circummediterranean Bivalve facies, cycles and Global sea level changes. In: II Congreso Geológico de España. Granada. 1 (Eds. J.P. Calvo \& C. Martín Escorza). Universidad de Granada y C.S.I.C. 342-344.

Hirsch, F., Márquez-Aliaga, A. y Santisteban, C. 1987. Distribución de Moluscos y Conodontos del Tramo superior del Muschelkalk en el sector occidental de la Provincia Sefardí. Cuadernos de Geología Ibérica, 11, 779-815.

Jiménez de Cisneros, D. 1929. Notas acerca del Triásico de Alicante. Asociación española para el progreso de las ciencias, $\mathbf{6}$, 37-39.

Llompart, C., Rosell, J., Márquez-Aliaga, A. y Goy, A. 1987. El Muschelkalk de la isla de Menorca, Cuadernos de Geología Ibérica, 11, 323-335.

López-Gomez, J. y Arche, A. 1992. Las Unidades Litoestratigráficas del Permico y Triásico Inferior y Medio en el Sector SE de la Cordillera Ibérica. Estudios Geológicos, 98, 123-143.

López-Gómez, J. y Arche, A. 1994. El Triásico y el Pérmico del SE de la Cordillera Ibérica. Guia de Campo, III Coloquio de Estratigrafía y Paleogeografía del Permico y Triásico de España. (Ed.A. Arche). Cuenca. 70 pp.

López-Gómez, J., Márquez-Aliaga, A., Arche, A. y Goy, A. 1987. La facies Muschelkalk de Henarejos (Cuenca): Sedimentología y fauna del tramo superior. Cuadernos de Geología Ibérica, 11, 665-676.

López-Gómez, J., Márquez-Aliaga, A., Calvet, F., Márquez, L. \& Arche, A. 1994. Aportaciones a la estratigrafía y paleontología del Triásico Medio de los alrededores de Agost, Alicante. Boletín de la Real Sociedad española de Historia Natural, 89 (1-4), 109-120.

López-Gómez, J., Mas, R. and Arche, A. 1993. Sequence stratigraphic analysis and paleogeographic interpretation of the Buntsandstein and Muschelkalk facies (Permo-Triassic) in the SE Iberian Ranges. Paleogeography, Palaeoclimatology, Palaeoecology, 103, 179-221.

Mallada, L. 1880. Sinopsis de las especies fósiles que se han encontrado en España. Sistema Triásico. Boletín de la Comisión del Mapa Geológico de España, 7, 241-256.

March, M. 1991. Los conodontos del Triásico Medio (Facies Muschelkalk) del noroeste de la Península Ibérica y de Menorca. Tesis doctoral. Universidad de Valencia. 375 pp.
Márquez, L. 1994. Los foraminíferos triásicos españoles: puesta a punto de los conocimientos existentes. Coloquios de Paleontología, 46, 75-88.

Márquez-Aliaga, A. 1976. Estudio paleontológico del Triásico Medio en el Sector Meridional de la Cordillera Ibérica. Tesina de Licenciatura. Madrid. 113 p.

Márquez-Aliaga, A. 1983. Aportación a la sistemática de los bivalvos del Triásico. Coloquios de Paleontología, 38, 33-47.

Márquez-Aliaga, A. 1985. Bivalvos del Triásico Medio del Sector Meridional de la Cordillera Ibérica y de los Catalánides. Publicaciones de la Universidad Complutense de Madrid, 40, 1-429.

Márquez-Aliaga, A., Arche, A., Brito, J., Calvet, F., Goy, A., López-Gómez, J. y Márquez, L. 1994. El Muschelkalk en el Anticlinal de Calanda (Teruel, España): Estratigrafía y contenido paleontológico. Boletín de la Real Sociedad española de Historia Natural, 89 (1-4), 145-166.

Márquez-Aliaga, A., Budurov, K. y Martínez, V. (En prensa). Registro de conodontos del Triásico Medio en un Nautiloideo de Espejeras (Alicante, España). Coloquios de Paleontología.

Márquez-Aliaga, A. y De Renzi, M. 1984. Temas viejos a la luz de nuevos conceptos: la paleontología del Triásico español. I Congreso Español de Geología. Segovia. 1, 451-460.

Márquez-Aliaga, A. y García-Gil, S. 1991. Paleontología y ambientes del Triásico Medio en el sector noroccidental de la Cordillera Ibérica. Estudios Geológicos, 47 (1-2), 85-95.

Márquez-Aliaga, A. and Hirsch, F. 1988. Migration of Middle Triassic Bivalves in the Sephardic Province. In: II Congreso Geológico de España. Granada. 1 (Eds. J.P. Calvo \& C. Martín Escorza). Universidad de Granada y C.S.I.C., 301-304.

Márquez-Aliaga, A., Hirsch, F. and López-Garrido, A. 1986. Middle Triassic Bivalves from the Hornos-Siles (Spain). Neues Jarhburch für Geologie und Paläontologie, 173 (2), 201-227.

Márquez-Aliaga, A. y López-Gómez, J. 1989. Sedimentología y Paleontología del Triásico Medio, Muschelkalk, de la Cordillera Ibérica. I: Cuenca y Valencia, España. Estudios Geológi$\cos , \mathbf{4 5}, 387-398$.

Márquez-Aliaga, A., Márquez, L., March, M., Goy, A. y Brito, J. M. 1987. Aspectos paleontológicos del Muschelkalk de Calanda. Cuadernos de Geología Ibérica, 11, 677-691.

Márquez-Aliaga, A. y Martínez, V. 1994. Procesos de colonización y su problemática, ejemplificada por bivalvos del Triásico español. Boletín de la Real Sociedad Española Historia Natural (Sección Geología), 89 (1-4), 121-130.

Márquez-Aliaga, A. y Montoya, P. 1991. El Triásico de Alicante: Un efecto Lázaro en los estudios paleontológicos. Revista Española de Paleontología, N $\mathbf{N}^{\mathbf{0}}$ Extra., 115-125.

Márquez-Aliaga, A., Santisteban, C. y Márquez, L. 1984. Triásico Medio de Bugarra (Valencia, España). Estudios Geológicos, 40, 365-374.

Martín-Algarra, A. 1987. Evolución geológica alpina del contacto entre las Zonas Internas y las Zonas Externas de la Cordillera Bética. Tesis Universidad de Granada. 1171 págs.

Martín-Algarra, A., Márquez-Aliaga, A., Solé de Porta, N. y Valenzuela, J. M. 1993. La serie triásica de Los Pastores (Algeciras). Estudios Geológicos, 49 (1-2), 1-20.

Martín-Algarra, A., Márquez-Aliaga, A. y Solé de Porta, N. 1994. El Trias Superior de los Taríquides y las relaciones entre los dominios de Facies Germánica y Alpina en la Cordillera Bética occidental. In: Resúmenes de las Comunicaciones, III Coloquio de Estratigrafía y Paleogeografía del Pérmico y Triásico de España. (Ed. A. Arche). Cuenca, 71-72. 
Martínez, V., Budurov, K. y Márquez-Aliaga, A. 1994. Hallazgo de conodontos triásicos en un ejemplar de Germanonautilus bidorsatus (SCHLOTHEIM, 1832) en el yacimiento de Espejeras (Alicante). In: Comunicaciones de las X Jornadas de Paleontología (Coord. S. Fernandez) Madrid, 128-130.

Pérez-Arlucea, M. 1992. Características de los sedimentos de la segunda transgresión del Triásico Medio (Ladiniense) en la zona central de la Cordillera Ibérica. Revista de la Sociedad Geológica de España, 4, 143-164.

Pérez-Arlucea, M. y Rey, D. 1994. Sedimentación continental y marina del Pérmico y Triásico en la zona central de la Cuenca Ibérica. Región de Albarracín-El Pobo de Dueñas. Guia de Campo, III Coloquio de Estratigrafía y Paleogeografía del Permico y Triásico de España. (Ed. A. Arche). Cuenca. 56 pp.

Pérez-Arlucea, M. y Sopeña, A. 1985. Estratigrafía del Pérmico y Triásico en el sector central de la Rama Castellana de la Cordillera Ibérica (provincias de Guadalajara y Teruel). Estudios geológicos, 41, 207-222.

Pérez-López, A. 1991. El Trias de facies germánica del sector central de la Cordillera Bética. Tesis Doctoral, Universidad de Granada. 400 pp.

Pérez-López, A., Fernández, J., Solé de Porta, M. y Márquez-Aliaga, A. 1991. Bioestratigrafía del Triásico de la zona Subbética (Cordillera Bética). Revista Española de Paleontología, $\mathbf{N}^{\mathbf{0}}$ Extra., 139-151.

Pérez-López, A. y Sanz de Galdeano, C. 1994. Tectónica de los materiales triásicos en el sector central de la zona Subbética (Cordillera Bética). Revista de la Sociedad Geológica de España, 7 (1-2), 141-153.

Pérez-López, A., Solé de Porta, M., Márquez, L. y Márquez-Aliaga, A. 1992. Caracterización y datación de una unidad carbonática de edad Noriense (Fm. Zamoranos) en el Trias de la zona Subbética. Revista de la Sociedad Geológica de España, 5 (12), 113-127.
Schmidt, M. 1935. Fossilien der Spanische Trias. Abhandlungen der Heidelberger Akademie der Wissenschaften, 22, 1-140.

Sopeña, A., Virgili, C., Arche, A., Ramos, A. y Hernando, S. 1983. El Triásico. In: Geología de España (Libro Jubilar J.M. Rios) Tomo II. (Ed. J. A. Comba). I.G.M.E., Madrid, 47-63 pp.

Sos, V. 1933. Los fósiles triásicos españoles del Museo Nacional de Ciencias Naturales de Madrid. Boletín de la Sociedad Española de Historia Natural, 33, 287-302.

Verneuil, M. M. de et Collomb, E. 1853. Coup d'oeil sur la constitution géologique de quelques provincies de l'Espagne. Bulletin de la Societe Geologique Francaise, 10 (2), 61-147.

Via, L. y Villalta, J.F. 1977. Paleontología y Paleoecología de los yacimientos fosilíferos del Muschelkalk Superior entre Alcover y Montral. Cuadernos de Geología Ibérica, 4, 247-258.

Vilaseca, S. 1920. Contribució al estudi dels terrenys triàsics de la provincia de Tarragona. Treballs del Musèu de Ciències Naturals de Barcelona, 8, 1-66.

Virgili, C. 1958. El triásico de los Catalánides. Boletín del Instituto Geológico y Minero de España, 69, 1-831.

Virgili, C., Sopeña, A., Ramos, A. y Hernando, S. 1977. Problemas de la cronoestratigrafía del Trias en España. Cuadernos de Geología Ibérica, 4, 57-88.

Würm, A. 1911. Untersuchungen über den Geologischen Bau und die Trias von Aragonien. Zeitschr. Deutsch. Geol. Gesellsch., 43, 37-175.

Würm, A. 1913. Beiträge zur Kenntnis der iberisch balearischen Triasprovinz. Verh-naturhist-med. Ver. Heidelberg. N. F., 12 (4), 477-599.
Manuscrito recibido: 12 de diciembre, 1994 Manuscrito aceptado: 2 de julio, 1995 